\title{
Rejection Sensitivity on Social Networking Sites: How It Differently Impacts Late Adolescents' Positive and Negative Affect
}

\author{
Liqin Liu ${ }^{\dagger}$, Ping $\mathrm{Ni}^{\dagger}$, Beibei Yang* \\ Department of Education, Xinyang College, Xinyang, China \\ Email address: \\ leshul@126.com (Beibei Yang) \\ ${ }^{*}$ Corresponding author \\ $\dagger$ Liqin Liu and Ping Ni are co-first authors.
}

To cite this article:

Liqin Liu, Ping Ni, Beibei Yang. Rejection Sensitivity on Social Networking Sites: How It Differently Impacts Late Adolescents' Positive and Negative Affect. Psychology and Behavioral Sciences. Vol. 10, No. 5, 2021, pp. 183-191. doi: 10.11648/j.pbs.20211005.14

Received: October 7, 2021; Accepted: October 25, 2021; Published: October 30, 2021

\begin{abstract}
Rejection sensitivity online was prevalent among adolescents that was potential risk factor for affect. The purpose of the study was to examine the relationship between rejection sensitivity on networking sites and sentimental responses through self-assesion and self-esteem respectively and the sequential mediated effect both of them, the research also tested the moderated role of gender. There were 823 adolescents included in this research. They completed a questionnaire packages consisting of 4 scales. The findings indicated that adolescents' emotional responses to rejection sensitivity on networking sites was significant, the mediating model was remarkable through self-assesion and significant sequential mediated effect both of self-assesion and self-esteem for affect among girls but not boys, however, rejection sensitivity on social networking sites was directly related to negative affect among boys, gender played a moderating role in the relationship between rejection sensitivity on social networking sites and positive affect/negative affect respectively, boys were sensitive to negative affect but girls were sensitive to both of positive and negative affect and boys responsed to stimulus slightly than girls. The findings provided a new insight to the prevention and intervention for the negative affect and improvement for positive affect among adolescents. The implication of the conclusions for understanding and counteracting rejection sensitivity on networking sites were discussed.
\end{abstract}

Keywords: Rejection Sensitivity on Social Networking, Self-Assesion, Self-Esteem, Positive and Negative Affect

\section{Introduction}

Social networking sites (SNS) has been popular among various groups, especially among adolescents, for instance, there was a social media account or social media platform from people to people such as "wei xin" and " $Q Q$ " or "friend circle" and "Qzone" in China. It was convenient for adolescents to keep contact with others both of their friends and nodding acquaintances even friends online through social networking sites. However, theirs risk perception for SNS was very low, so they were easy to be influenced by some negative events, when they felt rejection sensitivity on social networking sites (RSSNS), they would experience the sentimental responses to it such as positive and negative affect strongly (PA/NA). Williams [1] got the results that social exclusion threatened individuals' fundamental desires to sense of belong, which would evoke stress for the subjects [2]. The people who experienced higher level of rejection or exclusion would develope rejection sensitivity in later life.

\subsection{Rejection Sensitivity on Social Networking Sites and Positive and Negative Affect (PA/NA)}

According to the previous concept of rejection sensitivity (RS) [3], this research defined the RSSNS as feeling sensitivity produced by rejection on social networking sites. Just as the result demonstrated that RS threatened humans' belongings [4], which would make individuals overreact to ambiguous circumstances [5], which may create bad status for emotion. The present reviews indicated that the rejection sensitivity was a social phenomenon that potentially impacted many individuals in long-term and short-term. In this case, there was a increasing number of people examined the effects of RS on adolescents, all 
of which presented a same picture [6-8]. With higher level of the $\mathrm{RS}$, adolescents would overreact to negative events.

Recently, some researches have started paying attention to the relationship between rejection sensitivity and affect, for example, higher rejection sensitivity was associated with increased attention to sad faces [9], the increasing number of people realized that the effect of rejection sensitivity online to affect, there was a large body of people have begun to reveal that affect produced through rejection sensitivity was the cognitive risk factors of mental health such as suicide ideation [10], loneliness [11], decreased life satisfaction [12], depression [13] and social anxiety [14] and behaviors problems such as social avoidance and distress [15], aggression [16]. In the previous researches, the results demonstrated that appearance-based rejection was related to disordered eating, physical health, suicidal ideation [17, 18], thus they would experience negative affect such as depression, low self-esteem and social anxiety $[19,20]$. In the special circumstances when study and work online has become popular, increasing number of people were immersed in communicating online, but which may show shortcomings, for example, some people would feel rejection sensitivity by exclusion online and decreased well-being [5] and higher aggression [21], they were easy to suffer from negative affect that being short of facing-to-facing communication.

With the quick developing of social media, people's life was impacted deeply, to avoid these negative effect, the article was excepted to examine the cognitive model of rejection sensitivity on social networking sites, little was known about the effect of positive and negative affect. Thus the main purpose of the current study was to create a cognitive model of RSSNS on PA/NA. This was the first to evaluate the relationship between RSSNS and affect.

\subsection{The Mediated Role of Self-assesion (SA) and Self-esteem (SE)}

Here the "SA" was defined as self-evaluation, according to theory of self-worth [22], individuals pursued SA in order to avoid trauma of SE and manage fear and anxiety [23] or to make impression management to build good impression [24]. Many empirical researches suggested that SE would be threatened by self disabilities and body image [25], that was, individuals' SA may be impacted by others' feedback around setting, when they suffered from rejection on social networking sites, they would experience different level of SA through feedback, further, which may impact SE and affect, and others' appearance-based feedback would make individuals perceived risk of SE at the same time [26], which was similar to appearance rejection or appearance acceptance, that was, SA and SE may mediate the relation between RSSNS and affect, and SA and SE may play a sequentially mediated role.

\subsection{The Moderated Effect of Gender}

Though the RSSNS associated with affect, the degree of influences varied from girls to boys across situations in high school, that was, gender played a moderated role in the relationship between RSSNS and PA/NA. Some evidences suggested gender differences in the pathway [27], such as depression, well-being produced by appearance-based RS [28], these researches indicated that boys were less sensitive to RS than females [29]. But current researches mainly focused on females' sample, which may exist samples bias. Abdul [30] identified the moderating effect of gender for RS, in a word, perceived paternal rejection in childhood has influenced on both of adult males and females' RS, but maternal rejection didn't associated with both of adult males and females' RS. However, there was different voice, the result from a mixed sample revealed that there was neither significant differences on appearance-based RS for young girls nor boys [31] and RS didn't associate with mental outcomes such as depression [32]. From what mentioned above, the study drew the conclusion that gender may play a moderated role in the model of RSSNS.

\subsection{The Resent Study}

From mentioned above, firstly, the main goal of this research was to test the relationship between ordinary RSSNS and PA/NA, secondly, this study examined the mediating effect of SA/SE and sequential effect both of them between RSSNS and PA/NA, thirdly, the team evaluated the moderated role of gender.

\section{Method}

\subsection{Participants}

The study taken conveniences samples from public high school located in northern city in Shandong province in china, participants were permitted to give up and withdraw at any time. A total of 850 adolescents participated the research, 27 participants were excluded from the data owing to they didn't complete the survey, lastly, 823 subjects taken into the next analysis (577 females). The effected response rates was $96.82 \%$.

\subsection{Scales}

\subsubsection{Rejection Sensitivity on Social Networking Sites Scales (RSSNS)}

The scale was developed by Downey [33], which included 18 items, aiming at testing the intimate relationship, and have being revised by this research in order to fit better to measure the high school students' rejection sensitivity level on social networking sites and conform to the cultures in china. For example, the girlfriend/boyfriend was replaced by classmates/friends, the "You ask your parents for extra money to cover living expenses" was replaced by "You ask your parents for extra money to cover living expenses through mobile phone or social networking sites", and social networking sites was added to all items. The scale was rated by 5 -point Likert scale ( $1=$ very disagree, $5=$ very agree). The Cronbach's alpha was 0.806 in this study.

\subsubsection{Self Assesion (SA)}

The scale was developed by this research team according to Wright, which included 3 items, for example "how you asset yourself--excellent, medium, common", The Cronbach's alpha was 0.519 in this study. 


\subsubsection{Self Esteem (SE)}

The scale was developed by Rosenberg [34], which was one dimension, students rated the 10 items with 4-point Likert scale ( $1=$ not at all suit to me, $4=$ very suit to me), the higher of the total scores was, the higher of the self-esteem was, which were broadly used in previous study in china. The Cronbach's alpha was 0.836 in this research.

\subsubsection{Positive and Negative Affect Scale (PANAS)}

The scale was developed by Watson [35], which was revised in order to conform to this research, For the positive affect, before testing, $56 \%$ of the team worker (included 5 university teachers, 10 adolescents, 3 high school teachers) suggested canceled "alert, strong, attentive" so that suit better to high school students in China. The initial Cronbach's alpha was 0.86 of the questionnaire, this research was 0.915 . For the negative affect scale, through discussing, "scared, jittery" were excluded because of they were same with "afraid, nervous" in meaning, At the same, adding "gloomy" to the scale because of it was popular among adolescents in China. After revising, PANAS totally included 15 items, 5-point Likert scale was replaced by 6-point Likert scale because of making it more sensitive. The initial Cronbach's alpha was 0.87 of the questionnaire, this study was 0.863 .

\subsection{Data Analysis}

Firstly, descriptive analysis and pearson correlations were calculated among all adolescents, Secondly, The present study used PROCESS macro (Model 6) developed by Hayes [36] to test mediated effects for males' and females' adolescents respectively and the model effect. Thirdly, The present study used PROCESS macro (Model 59) developed by Hayes [36] to test moderated effects for the mediated model among females' adolescents, because the mediated model for males' adolescents was not significant, the moderated analysis was excluded by the system.

\section{Results}

\subsection{Descriptive and Correlation Analysis}

First of all, the research assessed whether all data were normally distributed. The values for skewness of 2.0 and higher and kurtosis of 7.0 and higher were considered to high non-normality, which impacted analysis results [37]. However, all of values were below the standards, which indicated that all data were normally distributed (see table 1).

Secondly, the research rated whether all variables were correlated or not. The results demonstrated that all correlation coefficients were significant. (see table 1).

Table 1. The correlations among cross rejection sensitivity in social networking sites model.

\begin{tabular}{llllll}
\hline variables & $\mathbf{1}$ & $\mathbf{2}$ & $\mathbf{3}$ & $\mathbf{4}$ & $\mathbf{5}$ \\
\hline 1. RSSNS & - & $0.141^{* *}$ & $-0.088^{*}$ & $-0.177^{* *}$ & $0.359^{* *}$ \\
2. SA & & - & $-0.360^{* *}$ & $-0.291^{* *}$ & $0.114^{* *}$ \\
3. SE & & & - & $0.395^{* *}$ & $-0.198^{* *}$ \\
4. PA & & & & - & $-0.301^{* *}$ \\
5. NA & & & & & - \\
M & 59.69 & 1.90 & 29.92 & 26.18 & 26.09 \\
SD & 10.28 & 0.67 & 4.58 & 8.04 & 8.69 \\
Skewness & -0.138 & 0.116 & -0.205 & 0.071 & 0.352 \\
Kurtosis & -0.049 & -0.756 & 1.000 & -0.595 & -0.642 \\
\hline
\end{tabular}

${ }^{* *} P<0.01,{ }^{*} P<0.05$

RSSNS $=$ rejection sensitivity in social networking sites $=1$; $\mathrm{SA}=$ self-assesion $=2 ; \mathrm{SE}=$ self-esteem $=3 ; \mathrm{PA}=$ positive affect $=4 ; \mathrm{NA}=$ negative affect $=5$.

\subsection{Mediated Effects}

The study rated the mediated effects for PA and NA among males' adolescents (see tables 2, 3). Firstly, the study test the mediated effect for PA among males' adolescents and controlled the class level (CL), residence location (RL) and childrens' number (CN). From table 2, the authors drew the conclusions that the directed effect from RSSNS to PA was not remarkable $(\beta=-0.015, P>0.05$, model 1$)$; the coefficient from RSSNS to SA was not remarkable $(\beta=-0.008, P>0.05$, model 2); the coefficient from RSSNS to SE was not significant $((\beta=0.066, P>0.05$, model 3$)$, and the relation between SA and SE was negatively significant $(\beta=-0.362$, $P<0.01$, model 3); when RSSNS, SA, SE together were added into the equation $(\mathrm{F}=8.233, P<0.001)$, only the $\mathrm{SE}$ positively associated with PA $((\beta=0.343, P<0.001$, model 4$)$. So the mediated model for PA among males' adolescents didn't remarkable.

Table 2. The mediated effect for PA among males'adolescents ( $N=246)$.

\begin{tabular}{|c|c|c|c|c|c|c|c|c|}
\hline \multirow{2}{*}{ predictors } & \multicolumn{2}{|c|}{ Model 1 (PA) } & \multicolumn{2}{|c|}{ Model 2 (SA) } & \multicolumn{2}{|c|}{ Model 3 (SE) } & \multicolumn{2}{|c|}{ Model 4 (PA) } \\
\hline & $\boldsymbol{\beta}$ & $\mathbf{t}$ & $\boldsymbol{\beta}$ & $\mathbf{t}$ & $\boldsymbol{\beta}$ & $\mathbf{t}$ & $\boldsymbol{\beta}$ & $\mathbf{t}$ \\
\hline CL & 0.059 & 0.905 & 0.017 & 0.261 & 0.013 & 0.209 & 0.059 & 0.981 \\
\hline RL & -0.089 & -1.263 & 0.064 & 0.908 & -0.040 & -0.603 & -0.059 & -0.920 \\
\hline $\mathrm{CN}$ & 0.025 & 0.355 & -0.020 & -0.286 & 0.032 & 0.495 & 0.009 & 0.137 \\
\hline RSSNS & -0.015 & -0.224 & -0.008 & -0.127 & 0.066 & 1.071 & -0.039 & -0.650 \\
\hline SA & & & & & $-0.362^{* *}$ & -6.027 & -0.119 & -1.882 \\
\hline SE & & & & & & & $0.343^{* * *}$ & 5.407 \\
\hline $\mathrm{R}^{2}$ & 0.011 & & 0.004 & & 0.138 & & 0.171 & \\
\hline $\mathrm{F}$ & 0.667 & & 0.229 & & $7.674^{* * *}$ & & $8.233^{* * *}$ & \\
\hline
\end{tabular}

Note: ${ }^{* * *} P<0.001,{ }^{* *} P<0.01,{ }^{*} P<0.05$

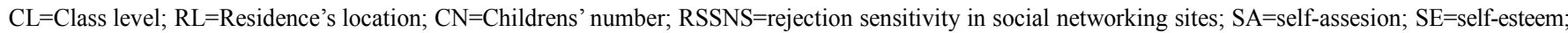
$\mathrm{PA}=$ positive affect; $\mathrm{NA}=$ negative affect; All 95\% confidence interval don't include 0 . 
Secondly, the study analysed the mediated effect for NA among males' adolescents (see table 3). From below table 3, when controlled the demographic variables, the team came to the following conclusions: the relationship between RSSNS and NA was positively significant $(\beta=0.380, P<0.001$, model $1)$; the RSSNS didn't associate with SA $(\beta=-0.008, P>0.05$, model 2); the RSSNS didn't associate with SE $(\beta=0.066$, $P>0.05$, model 3) but the coefficient from SA to SE was negatively significant $(\beta=-0.362, P<0.001)$ (model 3 ). Adding RSSNS, SA, SE to the structure equation, the model 4 was significant $(\mathrm{F}=11.625, P<0.001)$, the direct pathway coefficient from RSSNS $(\beta=0.339, P<0.001)$ and SE $(\beta=$ $-0.281, P<0.001)$ to NA was remarkable respectively, but the SA didn't predict NA $(\beta=-0.052, P>0.05)$ (model 4$)$. So the mediated model for NA among males' adolescents didn't significant.

Table 3. The mediated effect for $N A$ among males'adolescents $(N=246)$.

\begin{tabular}{|c|c|c|c|c|c|c|c|c|}
\hline \multirow{2}{*}{ predictors } & \multicolumn{2}{|c|}{ Model 1 (NA) } & \multicolumn{2}{|c|}{ Model 2 (SA) } & \multicolumn{2}{|c|}{ Model 3 (SE) } & \multicolumn{2}{|c|}{ Model 4 (NA) } \\
\hline & $\beta$ & t & $\beta$ & t & $\beta$ & $\mathbf{t}$ & $\beta$ & $\mathbf{t}$ \\
\hline CL & -0.056 & -0.934 & 0.017 & 0.261 & 0.013 & 0.209 & -0.053 & -0.924 \\
\hline RL & -0.003 & -0.048 & 0.064 & 0.908 & -0.040 & -0.603 & -0.017 & -0.279 \\
\hline $\mathrm{CN}$ & -0.060 & -0.928 & -0.020 & -0.286 & 0.032 & 0.495 & -0.050 & -0.802 \\
\hline RSSNS & $0.380^{* * *}$ & 6.275 & -0.008 & -0.127 & 0.066 & 1.071 & $0.399^{* * *}$ & 6.836 \\
\hline SA & & & & & $-0.362^{* * *}$ & -6.027 & -0.052 & -0.851 \\
\hline SE & & & & & & & $-0.281^{* * *}$ & -4.586 \\
\hline $\mathrm{F}$ & $11.079^{* * *}$ & & 0.229 & & $7.674^{* * *}$ & & $11.625^{* * *}$ & \\
\hline
\end{tabular}

Note: ${ }^{* * *} P<0.001,{ }^{* *} P<0.01,{ }^{*} P<0.05$

$\mathrm{CL}=$ Class level; $\mathrm{RL}=$ Residence's location; $\mathrm{CN}=$ Childrens' number; RSSNS=rejection sensitivity in social networking sites; $\mathrm{SA}=$ self-assesion; $\mathrm{SE}=$ self-esteem; $\mathrm{PA}=$ positive affect; $\mathrm{NA}=$ negative affect; All 95\% confidence interval don't include 0 .

Thirdly, the mediated effect for PA among females' adolescents was analysed (see table 4). From below table 4, After controlling demographic variables, the authors drew the following conclusions. The direct effect from RSSNS to PA was negatively significant $(\beta=-0.231, P<0.001$, model 1$)$; the relationship between RSSNS to SA was positively remarkable $(\beta=0.192, P<0.001$, model 2$)$; RSSNS $(\beta=-0.066$, $P>0.05)$ didn't associate with SE but the coefficient from SA $(\beta=-0.341, P<0.001)$ to $\mathrm{SE}$ was negatively remarkable (model 3). When all independent variables taken into the model 4 , the mediated model was significant $(\mathrm{F}=25.530$, $P<0.001)$, the pathway coefficient from RSSNS to PA was negatively remarkable $(\beta=-0.157, P<0.001)$, comparing with model 1, the coefficient decreased 0.074 from 0.231 to 0.157 (absolute value); SA negatively related with PA $(\beta=-0.170$, $P<0.001$ ), comparing with model 2 , the coefficient reduce 0.022 from 0.192 to 0.170 (absolute value); SE positively related with PA $(\beta=0.313, P<0.001)$. From mentioned above, it is clear that the direct effect from RSSNS to PA among females' adolescents was significant (model 1), the SA partly mediated the relationship between RSSNS and PA (the direct pathway coefficient lessened 0.074), the SE didn't mediate the model 1, but SA, SE sequentially mediated the relationship between RSSNS to PA (when SA, SE added into the model 4, the coefficient from SA to PA diminished 0.022 comparing with 0.170 ).

Table 4. The mediated effects for PA among females'adolescents ( $N=577)$.

\begin{tabular}{|c|c|c|c|c|c|c|c|c|}
\hline \multirow{2}{*}{ predictors } & \multicolumn{2}{|c|}{ Model 1 (PA) } & \multicolumn{2}{|c|}{ Model 2 (SA) } & \multicolumn{2}{|c|}{ Model 3 (SE) } & \multicolumn{2}{|c|}{ Model 4 (PA) } \\
\hline & $\boldsymbol{\beta}$ & $\mathbf{t}$ & $\boldsymbol{\beta}$ & $\mathbf{t}$ & $\boldsymbol{\beta}$ & t & $\boldsymbol{\beta}$ & $\mathbf{t}$ \\
\hline $\mathrm{CL}$ & -0.014 & -0.330 & 0.074 & 1.799 & $0.090^{*}$ & 2.288 & -0.021 & -0.561 \\
\hline $\mathrm{RL}$ & 0.033 & 0.710 & $0.121^{* *}$ & 2.652 & 0.059 & 1.348 & 0.048 & 1.129 \\
\hline $\mathrm{CN}$ & -0.029 & -0.626 & -0.015 & -0.330 & -0.085 & -1.960 & -0.006 & -0.143 \\
\hline RSSNS & $-0.231^{* * *}$ & -5.620 & $0.192^{* * *}$ & 4.677 & -0.066 & -1.641 & $-0.157^{* * *}$ & -4.092 \\
\hline SA & & & & & $-0.341^{* * *}$ & -8.559 & $-0.170^{* * *}$ & -4.194 \\
\hline SE & & & & & & & $0.313^{* * *}$ & 7.810 \\
\hline $\mathrm{F}$ & $8.281^{* * *}$ & & $8.022^{* * *}$ & & $18.671^{* * *}$ & & $25.530^{* * *}$ & \\
\hline
\end{tabular}

Note: ${ }^{* * *} P<0.001,{ }^{* *} P<0.01,{ }^{*} P<0.05$

$\mathrm{CL}=$ Class level; $\mathrm{RL}=$ Residence's location; $\mathrm{CN}=$ Childrens' number; $\mathrm{RSSNS}=$ rejection sensitivity in social networking sites; $\mathrm{SA}=\mathrm{self}$-assesion; $\mathrm{SE}=$ self-esteem; $\mathrm{PA}=$ positive affect; $\mathrm{NA}=$ negative affect; All 95\% confidence interval don't include 0 .

Lastly, the authors analysed the mediated model for NA among females' adolescents (see table 5). Here were the following results. After controlling demographic variables. The directed effect from RSSNS to NA was positively significant ( $\beta=0.353, P<0.001$, model 1); the relationship from RSSNS to SA was positively remarkable $(\beta=0.192, P<0.001$, model 2$)$;
RSSNS $(\beta=-0.066, P>0.05)$ didn't associate with SE but the coefficient from SA $(\beta=-0.341, P<0.001)$ to SE was negatively notable (model 3 ). When all independent variables taken into the model 4 , the mediated model was significant (F=17.316, $P<0.001$ ), the pathway coefficient from RSSNS to NA was positively remarkable $(\beta=0.327, P<0.001)$, comparing 
with model 1 , the coefficient decreased 0.026 from 0.353 to 0.327 (absolute value); SA didn't relate with NA $(\beta=0.061$, $P>0.05)$; SE negatively related with NA $(\beta=-0.109, P<0.01)$. From mentioned above, it is clear that the direct effect from RSSNS to NA among females' adolescents was significant (model 1), the SA didn't mediate the relationship between RSSNS and NA, the SE didn't mediate the model 1, but SA, SE sequentially mediated the relationship between RSSNS to NA (when SA, SE added into the model 4, the coefficient from SA to NA became insignificant.

Table 5. The mediated effects for NA among females' adolescents (N=577).

\begin{tabular}{|c|c|c|c|c|c|c|c|c|}
\hline \multirow{2}{*}{ predictors } & \multicolumn{2}{|c|}{ Model 1 (NA) } & \multicolumn{2}{|c|}{ Model 2 (SA) } & \multicolumn{2}{|c|}{ Model 3 (SE) } & \multicolumn{2}{|c|}{ Model 4 (NA) } \\
\hline & $\beta$ & t & $\beta$ & t & $\beta$ & $t$ & $\beta$ & t \\
\hline CL & -0.060 & -1.516 & 0.074 & 1.799 & $0.090^{*}$ & 2.288 & -0.057 & -1.454 \\
\hline $\mathrm{RL}$ & -0.005 & -0.116 & $0.121^{* *}$ & 2.652 & 0.059 & 1.348 & -0.011 & -0.241 \\
\hline $\mathrm{CN}$ & -0.048 & -1.093 & -0.015 & -0.330 & -0.085 & -1.960 & -0.055 & -1.280 \\
\hline RSSNS & $0.353^{* * *}$ & 8.990 & $0.192^{* * *}$ & 4.677 & -0.066 & -1.641 & $0.327^{* * *}$ & 8.232 \\
\hline SA & & & & & $-0.341^{* * *}$ & -8.559 & 0.061 & 1.445 \\
\hline SE & & & & & & & $-0.109^{* *}$ & -2.623 \\
\hline $\mathrm{F}$ & $22.294^{* * *}$ & & $8.022^{* * *}$ & & $18.671^{* * *}$ & & $17.316^{* * *}$ & \\
\hline
\end{tabular}

Note: ${ }^{* * *} P<0.001,{ }^{* *} P<0.01,{ }^{*} P<0.05$

$\mathrm{CL}=$ Class level; $\mathrm{RL}=$ Residence's location; $\mathrm{CN}=$ Childrens' number; RSSNS=rejection sensitivity in social networking sites; $\mathrm{SA}=$ self-assesion; $\mathrm{SE}=$ self-esteem; $\mathrm{PA}=$ positive affect; $\mathrm{NA}=$ negative affect; All 95\% confidence interval don't include 0 .

To demonstrate the mediated effect among females' adolescents, the bootstrap analysis was conducted (see table 6). As table 6 showed, the authors drew the following conclusions. The directed effect between RSSNS and PA was $(-0.231)$, the indirect effect for the pathway coefficient of RSSNS-SA-PA was $0.192 *(-0.170)=0.033$. The indirect effect for pathway coefficient of RSSNS-SA-SE-PA was $0.192 *(-0.341) * 0.313=0.020$. The indirect for pathway coefficient of RSSNS-SA-SE-NA was $0.192 *(-0.341) *(-0.109)=0.007$, all $95 \%$ CI of pathway coefficients didn't include 0 .

Table 6. Bootstrap analysis of mediated effects for affect among adolescents.

\begin{tabular}{|c|c|c|c|}
\hline \multirow{2}{*}{ Model pathways } & \multirow{2}{*}{ Females' samples $\beta$} & \multicolumn{2}{|c|}{$95 \%$ CI } \\
\hline & & lower & upper \\
\hline RSSNS-SA-PA & $0.192 *(-0.170)=0.033$ & -4.364 & -0.081 \\
\hline RSSNS-SA-SE-PA & $0.192 *(-0.341) * 0.313=0.020$ & -0.191 & -0.067 \\
\hline RSSNS-SA-SE-NA & $0.192 *(-0.341) *(-0.109)=0.007$ & -0.380 & -0.055 \\
\hline
\end{tabular}

Note: ${ }^{* * *} P<0.001,{ }^{* *} P<0.01,{ }^{*} P<0.05$

$\mathrm{CL}=$ Class level; $\mathrm{RL}=$ Residence's location; $\mathrm{CN}=$ Childrens' number; RSSNS=rejection sensitivity in social networking sites; $\mathrm{SA}=$ self-assesion; $\mathrm{SE}=$ self-esteem; $\mathrm{PA}=$ positive affect; $\mathrm{NA}=$ negative affect; All 95\% confidence interval don't include 0 ; bootstrap sample=1000.

\subsection{Moderated Mediated Effect of Gender}

As tables 2, 3, 4, 5 showed, the authors drew the conclusions, the mediated model from RSSNS to PA/NA were insignificant among males' adolescents but significant among females' adolescents, so the mediated model was more fit to females' adolescents, that was to say, gender played a moderated role in the mediated model.

\section{Discussion}

\subsection{The Directed Effect and the Moderated Role of Gender}

Just as the result shown, being in line with our hypothesis, the RSSNS was not related with PA among males' adolescents, but being positively associated with NA among males' adolescents, This was consistent with previous study [38], boys were less sensitive to RS-based appearance than girls. According to the results, the RSSNS made a difference for NA among males' adolescents, which was consistent with negative attention bias, that was, most of the people were sensitive about the negative stimuli [39], especially for males, the males' adolescents responded to negative (RSSNS) events more profoundly, so the level of NA was high. At the same time, the SA negatively related with SE for males' adolescents both for PA and NA., the higher self-assesion was, the more rigid they treated for themselves, and in turn, they would payed more attention to others' idea which produced the higher pressure imposed by others around surroundings, which was not in line with initial hypothesis that SA positively associated with SE. However, SE was positively related to PA among males, which further testified the negative relationship between SE and NA from the opposite side.

For the females' adolescents, there were a remarkable relationship between RSSNS and PA (negatively), NA (positively) respectively, the result was congruent with early results [40], Comparing with males' adolescents, females' adolescents responded to NA such as depression [41], which can be illustrated that females were more sentimental and emotional and they valued the interpersonal relationship than boys [42] so that they payed more attention to others' feedback 
such as appearance-based click and evaluation in "friend circle" or "Qzone" [43, 44], and emotional experiences was more fiercely than males no matter what experienced PA or NA, so when they felt social cues, for example, feeling sensitivity for rejection on social networking sites, the affect varied from PA and NA. The gender played a importantly moderated role. This was the first for us to examine the relationship between RSSNS and affect.

\subsection{The Mediated Role of Self-assesion and Self-esteem}

Being not consistent with the hypothesis, the mediated model was neither significant between RSSNS and PA nor that of NA through SA and SE or both of them for the sequential effect among males' adolescents, the result was partly confirm to prior research [45], for boys, they less cared others' evaluation about themselves and they expected slightly in certain situations, so SA/SE-based feedback may not employ its effect, psychological immunity offset the mediating effect, for example, appearance-based RS didn't correlate with depression, anger and SE [29], males were less impaired by negative cues in term of mental health and emotion [46], that was, the mediated model was wrong among males' adolescents, boys' emotion and affect fluctuation was less impacted by RSSNS, but the further effect needed to investigate whether mediated model was significant or not, because the males' samples of this research was less than females' sample.

The current study firstly test the mediating role of SA and the sequentially mediated effect both of SA and SE for model RSSNS-PA among females' adolescents. As shown above, SA partly mediated the relationship between RSSNS to PA and there was a significant sequentially mediating model between RSSNS and PA through SA and SE. SA negatively related to $\mathrm{SE}$ and $\mathrm{PA}$, which was inconsistent with initial hypothesis. When one was rejection online, they would feel risk for self-assesion to strengthen rejection sensitivity in turn according to self worth theory [22], because the interpersonal context was the important stressful sources [47], particularly on SNS where were short of body information and facial expression, the higher of SA was, the higher pressure around others' people online was. Though SA negatively predicted SE, but SE positively associated with PA (sequentially mediating), which could instruct girls to enhance positive affect experiences and decreased self expectation.

The mediating role of SA/SE didn't valid respectively, which didn't confirm to this hypothesis. Just as Jon demonstrated that females had a special fondness for passive cues in order to protect themselves [48], they were anxious and had initial negative affect, when they felt the higher level of risk factors such as rejection online in ambiguous situations, the passively emotional experience was produced fiercely, directly and quickly other than producing through SA or SE, in other word, negative affect directly was impacted by RSSNS based on initial NA, which may be determined by humans' physical characters and wouldn't be controlled by themselves. For the sequential mediating model, the higher RSSNS was, the higher SA was, in turn, they would felt higher self-esteem (SA-SE was positive), because the higher level of SA was, the higher exception was for girls for anythings in future, which produced higher self-esteem, when self-esteem was medium, it couldn't influenced positive affect, but when self-esteem was higher, that was another things that exceeded the proper level, females' adolescents would experience pressure produced by higher RSSNS through SA/SE, it was reasonable to deduce they would experience higher negative affect. So according to the research, the students should reduce anticipation for themselves and maintain medium self-esteem, just as a Chinese idiom goes "wu ji bi fan", in other word, when a thing reaches its extreme, it reverses its course. In China, the psychological phenomenon of "mian zi" was prevalent varying from people to people, so females' adolescents were just the one group of the most of people. When they was rejection online, they felt the danger of "broken mian zi" through the higher level of SA, SE, it was considerable that they would experience higher negative affects.

\section{Implications}

This study brought a new insight into the intervention and counteraction for the influences of RSSNS on affect. As mentioned above, the high school students' affect were easy impacted by RSSNS both of girls and boys, as a result, the school policy maker and teacher should focused on the NA of males' adolescents comparing with PA, of which impacted them profoundly, the teachers should teach them methods of emotional regulation to allay the NA. But for females' adolescents, the focus lied in enforcing PA and lessen NA, especially, in China, there were few sentimental management courses even none of that at all in high school because of the stress for entrancing to universities, all of time was consumed to study. It was urged to enhance the management through adding emotional course to fundamental teaching programs. Owing to RSSNS influenced affect differently for various gender, according to the theory of sex role socialization [49], of which, females were regarded as tender, careful, emotional but males were treated as strong, making bread, rational in China. So, females' adolescents were more influenced by rejection on social networking sites, they were sensitive to rejection online (PA, NA), males' adolescents were more immune to rejection sensitivity online comparing with females' adolescents, the courses should also be different, just as a old Chinese proverb saying "teach students in accordance with their aptitude". Secondly, parents and teachers should enhanced the risk perception for social media and strengthened the supervising and controlling for adolescents' using social networking sites (monitoring time and content online, eg, setting restrictions), teaching them correctly deal with stress produced by rejection online, properly regulated emotion varying from males and females' adolescents, which would decrease the bad behaviors and emotion [50]. Thirdly, cognitive empathy training should be offered for heightening gratitude and reduced bad emotion produced by bad behaviors [51]. 


\section{Conclusion}

First of all, the rejection sensitivity on social networking sites (RSSNS) was related with PA and NA among adolescents directly, that was, for the females' adolescents, RSSNS negatively related to PA and positively related to NA and RSSNS was positively related to SA; For the males' adolescents, RSSNS just only positively associated with NA and SA negatively associated with SE, SE positively associated with PA and negatively related to NA. Secondly, the mediated model of self-assessment and sequential mediated effect both of self-assessment and self-esteem were remarkable, that was to say, SA played a partly mediated role and SA, SE played a sequential mediated roles in the model of PA for females' adolescents; The SA, SE played a sequential mediated effect in the model of NA among females' adolescents. Thirdly, from what mentioned above, gender played a moderated role in the mediated model, girls were sensitive to positive affect and negative affect, boys were sensitive to negative affect, so the intervention programs for affect should be different.

\section{Funding Details}

This work was not supported by any funding.

\section{Authors' Contribution}

Liqin Liu: design, methods, data analysis, manuscript, paper review, revise;

Ping Ni: methods, data collection, data analysis;

Beibei Yang: methods, data analysis, revise

\section{Conflicts of Interest}

All the authors do not have any possible conflicts of interest.

\section{Acknowledgements}

Thanks all students who have participated in the investigations.

\section{References}

[1] Williams, K. D. (2007). Ostracism. Psychology, 58 (1), 425.

[2] Dickerson, S. S. \& Zoccola, P. M. (2013). Cortisol responses to social exclusion. In DeWall, C. N. (Ed): Oxford Handbook of Social Exclusion.

[3] Bowlby, J. (1969). Attachment and loss: Attachment. New York: Basic Books.

[4] Baumeister, R. F., \& Leary, M. R. (1995). The need to belong: Desire for interpersonal attachments as a fundamental human motivation. Psychological Bulletin, 117 (3), 497-529. http://dx.doi.org/10.1037/0033-2909.117.3.497

[5] Kimberly, J. B., \& Diane, M. Q. (2016). Weight-based rejection sensitivity: Scale development and implications for well-being. Body Image, 16 , http://dx.doi.org/10.1016/j.bodyim.2015.11.005

79-92.

[6] Charlotte, R., \& Babette, R. (2014). Rejection sensitivity as a mediator of the relationship between experienced rejection and borderline characteristics. Personality and Individual Differences 69, 176-181. http://dx.doi.org/10.1016/j.paid.2014.05.032

[7] Melanie, J. Z. (2015). Emotional sensitivity before and after coping with rejection: A longitudinal study. Journal of Applied Developmental Psychology, 41, 28-37. http://dx.doi.org/10.1016/j.appdev.2015.05.001

[8] Julie, L. P., Tracy, D., Alex, B., Beth, G., \& Janelle, S. (2019). Partner self-esteem and interpersonal risk: Rejection from a low self-esteem partner constrains connection and increases depletion. Journal of Experimental Social Psychology 80, 1730. https://doi.org/10.1016/j.jesp.2018.09.006

[9] Morganne, A. K., Lucas J. A., \& Tony, T. W. (2018). Rejection sensitivity, interpersonal rejection, and attention for emotional facial expressions. Journal of Behavior Therapy and Experimental Psychiatry, 59, 31-39. https://doi.org/10.1016/j.jbtep.2017.11.004

[10] Sarah, L. B., Sean, M. M., Jared, F. R., Nikki L. L., \& Kelly, C. C. (2019). Rejection sensitivity and suicide ideation among psychiatric inpatients: An integration of two theoretical models. Psychiatry Research, 272, 54-60. https://doi.org/10.1016/j.psychres.2018.12.009

[11] Rebecca, N., Rebecca, T., \& Pamela, Q. (2018). Influence of loneliness and rejection sensitivity on threat sensitivity in romantic relationships in young and middle-aged adults. Personality and Individual Differences, 131 (1), 185-190. https://doi.org/10.1016/j.paid.2018.04.047

[12] Nagila, K., Donna, A. M., Marry, S., \& Marcel, A. G. (2018). How borderline personality characteristics affect adolescents' life satisfaction: The role of rejection sensitivity and social relations. European Journal of Developmental Psychology, 15 (5), 594-607. https://doi.org/10.1080/17405629.2017.1321983

[13] Gao, S., Assink, M., Cipriani, A., \& Lin, K. (2017). Associations between rejection sensitivity and mental health outcomes: A meta-analytic review. Clinical Psychology Review, 57, 59-74. https://doi.org/10.1016/j.cpr.2017.08.007

[14] Angel, F., Anu, A., Cassidy, G., Courtney, C., Sabine, W., Stefan, G. \& Hofmann.(2011). Rejection sensitivity mediates the relationship between social anxiety and body dysmorphic concerns. Journal of Anxiety Disorders, 25, 946-949. doi: 10.1016/j.janxdis.2011.06.001.

[15] John, W., Dtrew, \& Nesdale. (2012). Rejection Sensitivity, Social Withdrawal, and Loneliness in Young Adults. Journal of Applied Social Psychology, 42 (8), 1984-2005. doi: 10.1111/j.1559-1816.2012.00927.x.

[16] Chopik, W. J., \& Giasson, H. L. (2017). Age differences in explicit and implicit age attitudes across the life span. The Gerontologist, $\quad 57 \quad$ (2), $169-177$. https://academic.oup.com/gerontologist/article-abstract/57/sup pl_2/S169/3913364

[17] Thompson, I., Hong, J. S., Lee, J. M., Prys, N. A., Morgan, J. T., \& Udo-Inyang, I. (2020). A review of the empirical research on weight-based bullying and peer victimisation published between 2006 and 2016. Educational Review, 72 (1), 82-110. https://doi.org/10.1080/00131911.2018.1483894 
[18] Ford, R., King, T., Priest, N., \& Kavanagh, A. (2017). Bullying and mental health and suicidal behaviour among 14-to 15-year-olds in a representative sample of Australian children. Australian New Zealand Journal of Psychiatry. 51 (9), 897-908. https://doi.org/10.1177/0004867417700275

[19] Schaan, V. K., Schulz, A., Bernstein, M., Schachinger, H., \& Vogele, C. (2020). Effects of rejection intensity and rejection sensitivity on social approach behavior in women. PLOS ONE, $15 \quad$ (1): $\quad$ e227799. https://doi.org/10.1371/journal.pone.0227799

[20] Bernstein, M. J., \& Claypool, H. M. (2012). Social Exclusion and Pain Sensitivity: Why Exclusion Sometimes Hurts and Sometimes Numbs. Personality and Social Psychology Bulletin, $38 \quad$ (2), 185-196. https://doi.org/10.1177/ 0146167211422449 PMID: 21885860

[21] Anna, M. M., \& Gemma, R. (2018). Rejection Sensitivity, Jealousy, and the Relationship to Interpersonal Aggression. Journal of Interpersonal Violence, 33 (13), 2118-2129. doi: 10.1177/0886260515622572.

[22] Wright, B. A. (1960). Physical disability: A psychological approach. New York: Harper \& Row.

[23] Crocker, J., \& Park, L. E. (2004). The costly pursuit of self-esteem. Psychological Bulletin, 130 (4), 392-414. https://doi.org/10.1037/0033-2909.130.3.392

[24] Hong, C., Chen, Z., \& Li, C. (2017). "Liking" and being "liked": How are personality traits and demographics associated with giving and receiving "likes" on Facebook? Computers in Human Behavior, 68: 292-299. https://doi.org/10.1016/j.chb.2016.11.048

[25] Porch, T. (2015). Society, Culture, and the Selfie: Analysis of the Impact of the Selfie Practice on Women's Body Image. Atlanta, GA: Emory University.

[26] Tylka, T. L., \& Wood-Barcalow, N. L. (2015a). The Body Appreciation Scale-2: Item refinement and psychometric evaluation. Body Image, 12, 53-67. http://dx.doi.org/10.1016/j.bodyim.2014.09.006

[27] Vogt Yuan, A. S. (2010). Body perceptions, weight control behavior, and changes in adolescents' psychological well-being over time: a longitudinal examination of gender. Youth Adolescents, 39, 927-939. https://link.springer.com/article/10.1007\%2Fs10964-009-9428 $-6$

[28] Hyde, J. S., Mezulis, A. H., \& Abramson, L. Y. (2008). The $\mathrm{ABCs}$ of depression: integrating affective, biological, and cognitive models to explain the emergence of the gender difference in depression. Psychology Review. 115, 291-313. doi: 10.1037/ 0033-295X.115.2.291.

[29] Schmidt, J., \& Martin, A. (2019). Appearance Teasing and Mental Health: Gender Differences and Mediation Effects of Appearance-Based Rejection Sensitivity and Dysmorphic Concerns. Frontiers in Psychology, 10, 579. doi: 10.3389/fpsyg.2019.00579.

[30] Abdul, K., Muhammad, K. U., Kazi, N. H., Md, N. S., \& Anjuman, S. (2019). Perceived Parental Acceptance-Rejection in Childhood Predict Psychological Adjustment and Rejection Sensitivity in Adulthood. Psychology Study 64 (4), 447-454. https://doi.org/10.1007/s12646-019-00508-Z

[31] Webb, H. J., Zimmer-Gembeck, M. J., Waters, A. M., Farrell,
L. J., Nesdale, D., \& Downey, G. (2017). Pretty Pressure From Peers, Parents, and the Media: A Longitudinal Study of Appearance-Based Rejection Sensitivity. Journal of Research $\begin{array}{llll}\text { on } \quad \text { Adolescence, } 27 & \text { (4), }\end{array}$ https://doi.org/10.1111/jora.12310

[32] Shuling, G., Mark, K., Andrea, C., \& Kangguang, L. (2017). Associations between rejection sensitivity and mental health outcomes: A meta-analytic review. Clinical Psychology Review, 57, 59-74. https://doi.org/10.1016/j.cpr.2017.08.007

[33] Downey, G., \& Feldman, S. I. (1996). Implications of rejection sensitivity for intimate relationships. Journal of Personality and Social Psychology, 70 (6), 1327-1343. http://dx.doi.org/10.1037/0022-3514.70.6.1327

[34] Rosenberg, F. (1995). Global self-esteem and specific self-esteem: Different concepts, Different outcomes. American Sociological Review, 60 (1), 141-156. https://www.jstor.org/stable/2096350

[35] Watson, D., Clark, L. A., \& Tellegen, A. (1988). Development and validation of brief measures of Positive and Negative Affect: The PANAS scales. Journal of Personality and Social Psychology, $54 \quad$ (6), $1063-1070 . \quad$ doi: 10.1037/0022-3514.54.6.1063.

[36] Hayes, A. F. (2013). Introduction to mediation, moderation, and conditional process analysis: A regression-based approach New York: Guilford Press.

[37] Muthén, B., \& Kaplan, D. (1992). A comparison of some methodologies for the factor analysis of non-normal Likert variables: A note on the size of the model. The British Journal of Mathematical and Statistical Psychology, 45, 19-30. http://dx.doi.org/10.1111/j.2044-8317.1992.tb00975.x.

[38] Stubbs-Richardson, M., Sinclair, H. C., Goldberg, R. M., Ellithorpe, C. N., \& Amadi, S. C. (2018). Reaching out versus lashing out: examining gender differences in experiences with and responses to bullying in high school. American Journal of Criminal Justice, 43, 39-66. doi: 10.1007/s12103-0179408-4.

[39] Yue, L., Xing, Q., \& Yanmei, Z. (2018). Modulation of Brain Response to Peer Rejection by Rejection Sensitivity: An Exploratory Study, Neuropsychologia, 117, 389-397. https://doi.org/10.1016/j.neuropsychologia.2018.07.003

[40] Zimmer-Gembeck, M. J., Nesdale, D., Webb, H. J., Khatibi, M., \& Downey, G. (2016). A longitudinal rejection sensitivity model of depression and aggression: unique roles of anxiety, anger, blame, withdrawal, and retribution. Abnormal Children Psychology, 4, 1291-1307. doi: 10.1007/s10802-016-0127-y.

[41] Salk, R. H., Petersen, J. L., Abramson, L. Y., \& Hyde, J. S. (2016). The contemporary face of gender differences and similarities in depression throughout adolescence: development and chronicity. Journal of Affective Disorders, 205, 28-35. http://dx.doi.org/10.1016/j.jad.2016.03.071

[42] Malti, T., Buchmann, M. (2010). Socialization and individual antecedents of adolescents' and young adults' moral motivation. Journal of Youth Adolescents. 39, 138-149. doi: 10.1007/s10964-009-9400-5.

[43] Chua, T. H. H., \& Chang, L. (2016). Follow me and like my beautiful selfies: Singapore teenage girls' engagement in self-presentation and peer comparison on social media. Computers in Human Behavior, 55 (A), 190-197. https://doi.org/10.1016/j.chb.2015.09.011 
[44] Bazarova, N. N. \& Choi, Y. H. (2014). Self-disclosure in social media: Extending the functional approach to disclosure motivations and characteristics on social network sites. Journal of Communication, 64 (4), 635-657. https://doi.org/10.1111/jcom.12106

[45] Bleidorn, W., Arslan, R. C., Denissen, J. J., Rentfrow, P. J., Gebauer, J. E., Potter, J., et al. (2016). Age and gender differences in self-esteem-A cross-cultural window. Journal of Personality Social Psychology, 111 (3), 396-410. http://dx.doi.org/10.1037/pspp0000078

[46] Von Soest, T., Wichstrøm, L., \& Kvalem, I. L. (2016). The development of global and domain-specific self-esteem from age 13 to 31. Journal of Personality and Social Psychology, 110 (4), 592-608. http://dx.doi.org/10.1037/pspp0000060

[47] Teo, A. R., Choi, H., \& Valenstein, M. (2013). Social relationships and depression: ten-year follow-up from a nationally representative study. PLOS ONE, 8 (4), e62396. doi: 10.1371/journal.pone.0062396.
[48] Jon, D. E., Dmitri, R., Liu, T., \& Yang, H. B. (2020). Fear of missing out predicts repeated measurements of greater negative affect using experience sampling methodology. Journal of Affective Disorders, 262 (1), 298-303. https://doi.org/10.1016/j.jad.2019.11.026

[49] Williams, J. A., Vernon, J., Williams, M. C., \& Malecha, K. (1987). Sex Role Socialization in picture books: an update. Sociology Department, Faculty Publications, 68. (1), 148-156, https://digitalcommons.unl.edu/sociologyfacpub/8

[50] Symons, K., Vanw, E. I., Walrave, M., Van, O. J., \& Ponnet, K (2019). Parents' concerns over internet use, their engagement in interaction restrictions, and adolescents' behavior on social networking sites. Youth and society, 52 (8), 1569-1581. https://doi.org/10.1177/0044118X19834769

[51] Ibrahim, T., Özgür, E. (2019). Motives Behind Cyberbullying Perpetration: A Test of Uses and Gratifications Theory. Journal of Interpersonal Violence, 1-26. doi: $10.1177 / 0886260518819882$. 